\title{
Design of Far-red Fluorescent Kv1.3 Channel for Membrane Expression in Eukaryotic Cells and Its Interactions with Hongotoxin1
}

Nikita Orlov $^{1}$, Anastasia Ignatova ${ }^{2}$, Oksana Nekrasova ${ }^{1}$, Mikhail Kirpichnikov ${ }^{1}$ and Alexey Feofanov ${ }^{1}$

${ }^{1}$ Lomonosov Moscow State University, Shemyakin-Ovchinnikov Institute of Bioorganic Chemistry, Vladimir, Vladimir, Russia, ${ }^{2}$ Shemyakin-Ovchinnikov Institute of Bioorganic Chemistry of the Russian Academy of Sciences, Moscow, Moskva, Russia

Potassium voltage-gated Kv1.3 channels found in many cell types of the nervous and immune systems control membrane potential and regulate a lot of cellular functions such as proliferation, migration, cytokine secretion, and apoptosis. Activity and elevated expression level of Kv1.3 channels mediate proinflammatory response in auto-immune disorders (rheumatoid arthritis, Type 1 diabetes, and asthma), and neuroinflammatory diseases (multiple sclerosis, ischemic stroke, Alzheimer's disease, and epilepsy) [1]. Inhibition of Kv1.3 channels activity in many cases resulted in the anti-inflammatory effect and is considered as a prospective therapeutic strategy. Peptide toxins from the scorpion venom with their ability to bind specifically and with high affinity to the target Kv1 channels represent a rich source of Kv1.3channel blockers and are considered as promising templates for drug development [2].

To facilitate development of Kv1.3-targeted drugs and study of their properties at the cellular level, we have designed a chimeric protein mKate2-Kv1.3, which has enhanced plasma membrane expression in eukaryotic cells and fluoresces due to using a very bright far-red fluorescent protein mKate2. Plasmid encoding mKate2-Kv1.3 was obtained by cloning a KCNA3 gene between BglII and HindIII sites of the pmKate2-C vector (Evrogen, RF). mKate 2 was linked to the N-terminus of the Kv1.3 channel through the 9-aa flexible linker. The procedure of HEK293 cells transfection with the plasmid pmKate2-Kv1.3 was optimized to achieve transient expression of mKate2-Kv1.3 in more than $40 \%$ of cells. Confocal laser scanning microscopy analysis revealed that mKate2-Kv1.3 accumulates in both cytoplasmic cellular structures and plasma membrane (Fig. 1, B, F). To investigate the state of mKate2-Kv1.3 in plasma membrane we have applied a high affinity fluorescent ligand A-HgTx1 (HgTx1 labeled with Atto488), which was characterized by us previously [3]. A-HgTx1 is found to bind to plasma membrane of those cells, which demonstrate membrane expression of mKate2-Kv1.3 (Fig. 1, A), thus indicating to formation of A-HgTx1-Kv1.3 complexes. No intracellular accumulation of A-HgTx1 is observed at the used conditions. A-HgTx1 binding to Kv1.3 is detected at the A-HgTx1 concentration of tens nmole/l and proves tetramerization of Kv1.3 and correct formation of the pore blocker outer binding site situated in the pore domain of the channel. Specificity of this binding was demonstrated in the competitive binding experiment, when an excess of HgTx 1 displaced totally A-HgTx1 from the complexes with Kv1.3 at the cell membrane (Fig. 1, E-H). A-HgTx1 binding to Kv1.3 at the plasma membrane can be realized at a variable composition of cell medium that open a way to study influence of various factors (including proteins and concentration of different ions) on blocker-channel interactions.

Combination of HEK293 cells expressing fluorescent $\mathrm{K}_{\mathrm{v}} 1.3$ at the plasma membrane and A-HgTx 1 as a fluorescent ligand was concluded to be a promising platform for search and study of pharmacologically relevant channel blockers by using competitive binding approach as well as the features of channel expression, traffic, distribution and functioning [4]. 

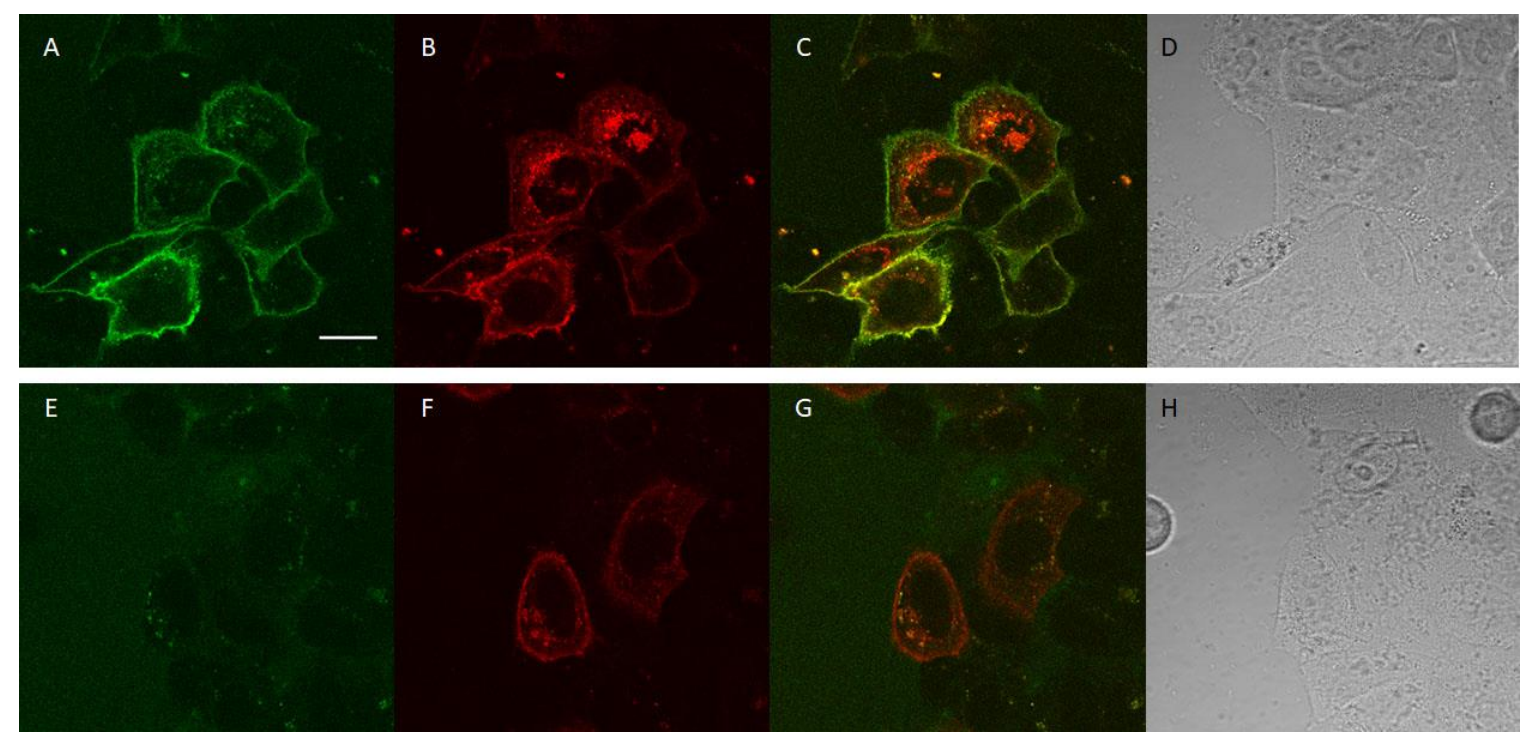

Figure 1. CLSM analysis of interactions of peptide pore blocker HgTx1 with mKate2-Kv1.3 channels, transiently expressed in HEK293 cells. (A-D) mKate2-Kv1.3 expressing cells stained with $40 \mathrm{nM}$ AHgTx1. (E-H) Displacement of A-HgTx1 (40 nM) from the complexes with mKate2-Kv1.3 by HgTx1 $(400 \mathrm{nM})$. (A,E) Fluorescent images showing distribution of A-HgTx1. (B, F) Fluorescent images showing distribution of mKate2-Kv1.3. (C, G) Merged images of A-HgTx1 and mKate2-Kv1.3 distributions. (D, H) Transmitted light images of cells. Bar - $20 \mathrm{~mm}$.

\section{References}

[1] M Pérez-Verdaguer et al. Expert Opin Ther Targets. 20 (2016), p.577-591.

[2] S Peigneur et al. Toxins (Basel). 10 (2018), pii: E126.

[3] N Orlov, O Nekrasova, A Feofanov Microsc. Microanal. 25(S2) (2019), p. 1278-1279.

[4] Financial support of the Program of Presidium of Russian Academy of Sciences is acknowledged. 